\title{
Perception of Animacy in Caucasian and Indian Faces
}

\author{
Aleksandra Swiderska*,Eva Krumhuber, Arvid Kappas \\ Jacobs University Bremen
}

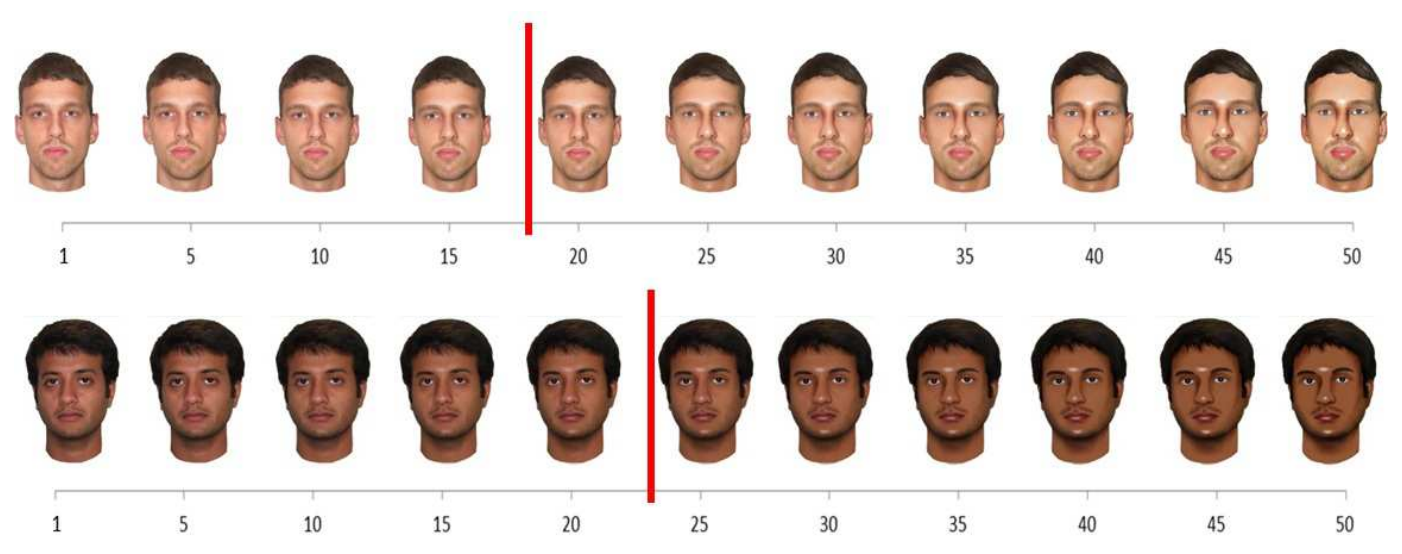

Figure 1: The faces' continua from real (1) to artificial (50) face. Animacy boundary is marked in red (mean number of face perceived as no longer alive: 18.70 for Caucasians and 22.57 for Indians, $p<.001$.

\section{Introduction}

Masahiro Mori, who introduced the concept of the Uncanny Valley, recommended settling for moderate levels of human likeness in robotic design in order to avoid the eeriness upon encountering entities closely resembling humans [Mori 1970]. However, the strive for highly humanlike design continues, nowadays particularly in areas such as computer graphics and animation, inspiring research on what actually is and what is not perceived as a human being.

Using real and computer-generated faces as well as a range of morphs between the two, it can be demonstrated that at some point along a continuum of morphs between a completely human and completely artificial face, people stop attributing aliveness to the face even if the general appearance of the artificial face perfectly matches that of the real face. This point is referred to as the animacy boundary and has been shown to fall rather close to the human endpoint of the continuum [Looser and Wheatley 2010].

\section{Our Approach}

Based on psychological theories on intergroup perception and prejudice (e.g., [Harris and Fiske 2006]), it may be expected that people's attribution of aliveness to faces would depend on group membership that the faces indicate. The purpose of the present research was therefore to investigate whether the perception of the animacy boundary would be influenced by the ethnicity of the face. Specifically, we hypothesized that the point at which the animacy boundary is detected would differ for Caucasian and Indian faces, as evaluated by Caucasian participants.

60 Caucasian students at Warsaw University, Poland, participated in this study. Materials comprised photographs of neutral faces of 10 Caucasian and 10 Indian men. Additionally, a series of 48 morphs between each of these faces and their artificial analogues were created. The analogues were produced via modifying the surface of the skin, eyes, and hair of the real faces from the photographs (facial morphology was preserved). Thus, this added up to 50 versions of each face (real face, 48 morphs, and artificial face).

Participants viewed either the Caucasian or Indian faces' continua beginning at the real endpoint and progressing through the morphs until the artificial endpoint. Their task was to determine the point at which the faces did not look alive anymore. That is, they were to find the perceived animacy boundary (see Figure 1).

\section{Results}

The results showed a significant difference in where the animacy boundary was perceived depending on the faces' ethnicity. Participants evaluated the animacy boundary for Caucasian faces to fall closer to the human endpoint of the morphs' continua in comparison to Indian faces (see Figure 1). Work in the area of animation has been focused mainly on increasing the realism of computergenerated faces but this finding suggests that it might be also important to take factors such as the ethnicity of both viewers and characters into account while developing humanlike design of animated characters. Thus, psychological processes play an important role in how alive a face appears to be and require consideration in fields of computer graphics and human-computer interaction (HCI).

Acknowledgments This work has been supported by the European Commission project eCUTE - Education in Cultural Understanding, Technologically-Enhanced (FP7-ICT-257666).

\section{References}

HarRis, L. T., AND FISKE, S. T. 2006. Dehumanizing the lowest of the low: Neuroimaging responses to extreme outgroups. Psychological Science 17, 847-853.

Looser, C. E., And Wheatley, T. 2010. The tipping point of animacy: How, when and where we perceive life in a face. Psychological Science 21, 1-9.

MORI, M. 1970. The uncanny valley. Energy 7, 33-35.

\footnotetext{
*e-mail: a.swiderska@jacobs-university.de
} 